\title{
ESCLEROSE TUBEROSA
}

\author{
ESTUDO MULTIDISCIPLINAR DE 15 CASOS
}
E. M. T. YACUBIAN *
L. C. COLARILLE ***
T. R. LAUANDOS *****
F. B. ASSUMPCAO JR. **
M. MARCUCCI **
J. C. DUARTE **
M. F. L. MADRUGA ****
L. M. DA CRUZ ***
M. H. SPROVIERI ****
T. C. L. RAMOS *****
S. A. FUUARI *******

A esclerose tuberosa, incluída no grupo das facomatoses - também referida como doença de Pringle, doença de Bourneville, adenoma sebáceo e epilóia - caracteriza-se pelo crescimento anormal de células de origem ectodérmica e mesodérmica, levando ao aparecimento de quadro clínico variável, cujas principais manifestações incluem deficiência mental, epilepsia e tumores, mais comumente na face, cérebro, coração e rim. A primeira descrição da doença, caracterizando as lesões cutâneas faciais com sua típica distribuição naso-labial, data ainda do século passado (1835) e é de autoria do dermatologista francês Rayer, citado por Morgan e Wolfort ${ }^{13}$ em seu trabalho de revisão de 1979. Este mesmo trabalho traça um histórico das descobertas referentes à esclerose tuberosa. Assim, von Recklinghausen (1862) descreveu um tumor cardíaco encontrado na necrópsia de um recém-nascidn, sendo o primeiro a descrever as características microscópicas da esclerose tuberosa. Em 1880, Bourneville relatou o caso de menina que apresentava deficiência mental, hemiplegia $e$ epilepsia; na necrópsia, foram encontrados tumores no cérebro e nos rins; Bourneville acreditava que os tumores cerebrais eram a causa de suas crises convulsivas e do retardo mental. Balzer e Ménétrier (1885) descreveram o caso de uma paciente cujas lesões cutâneas foram interpretadas como adenoma sebáceo. Em 1890, Pringle foi o primeiro a descrever as características microscópicas das lesões faciais, e em 1895, Besnier reconheceu a esclerose tuberosa como doença sistêmica e não distúrbio raro limitado à pele. Crocker (1903) corretamente referiu que o adenoma sebáceo era anormalidade de vários elementos dérmicos, incluindo o tecido conjuntivo, glândulas sebáceas, folículos pilosos, glândulas sudoriparas e capilares. Campbell (1905) descreveu a tríade de achados clínicos epilepsia, idiotia e adenoma sebáceo, que ele acreditava ser conclusiva para o diagnóstico. Em 1911, Sherlock introduziu o termo "epilóia" para indicar a tríade clínica (epilepsia, baixo nivel intelectual e adenoma sebáceo).

$O$ presente trabalho apresenta os sinais e sintomas observados numa amostra de 15 casos estudados por uma equipe multidisciplinar, visando a

Trabalho da APAE de São Paulo: * neuropediatra; ** psiquiatra; *** psicóloga; **** assistente social; **** fonoaudióloga; ***** geneticista; ****** pediatra. 
contribuir para o melhor conhecimento desta doença que é uma das mais graves entre as facomatoses, levando a sérios danos ao SNC.

\section{MATERIAL E METODOS}

Neste estudo, relatamos 15 casos de esclerose tuberosa diagnosticados no Ambulatório do Centro de Habilitação da APAE de São Paulo, entre os anos de 1967 e 1982. Todos os casos foram submetidos à rotina básica do Centro por equipe multidisciplinar, incluindo estudo social, avaliação pediátrica, exame neurológico, exame psiquiátrico, avaliação psicológica, avaliação fonoaudiológica, estudo genético e estudo laboratoria] (para pesquisa de erros metabólicos).

O diagnóstico foi baseado na existência de um ou mais dos seguintes achados: adenoma sebáceo, manchas hipocrômicas, fibromas periungueais, deficiência mental, epilepsia, facomas retinianos e presença de calcificações intracranianas, evidenciadas pelo estudo radiológico convencional ou tomografia axial computadorizada do crânio. Em 50\% dos casos devido à impossibilidade de seguimento dos pacientes, os dados foram obtidos através dos prontuários. Todos os pacientes foram submetidos a avaliação psicológica, cujo objetivo foi a verificação do nível intelectual. Dos 15 casos, 2 foram submetidos à Escala de Desenvolvimento de Gesell, 3 à Escala de Terman-Merril forma M, 4 à Escala de Inteligência para Crianças (WISC). Em 1 caso, como não se conseguiu avaliação precisa do nível intelectual, foi utilizada a Escala de Maturidade Social de Vineland. Nos demais casos, rã̃o foi possível avaliação precisa do nível mediante provas, sendo a classificação obtida através da observação direta do paciente. A anamnese foi dirigida visando a deteção de sinais de esclerose tuberosa em outros membros da família.

\section{RESULTADOS}

Composição da amostra (Tabela 1) - Dos 15 casos, 8 eram do sexo masculino e 7 do sexo feminino. Todos eram da raça branca. A faixa etária por ocasião da primeira consulta variava de 1 ano e 7 meses até 23 anos. Quanto à herança, 11 casos (73\%) foram considerados esporádicos e 4 não puderam ser classificados.

Sinais cutaneos (Tabela 2) - O adenoma sebáceo estava presente em 9 casos (60\%). Manchas hipocrômicas, tendo algumas o característico aspecto foliáceo, foram observadas em 9 casos (60\%). Outras lesões de pele, como manchas café-com-leite e nódulos cutâneos estavam presentes em 6 casos (40\%). Fibromas periungueais foram encontrados em apenas 3 casos (20\%).

Sintomatologia neuropsiquiátrica - A tabela 3 especifica o nível intelectual, ressaltando 0 fato de que apenas 2 pacientes tinham inteligência limitrofe, enquanto todos os outros apresentavam graus variáveis de deficiência mental. Na mesma tabela são precisados os diferentes tipos de crises epilépticas, presentes em 13 (86\%) dos 15 casos, e os distúrbios psiquiátricos, como a hiperatividade e agressividade, presentes em 9 casos (60\%). 
Composição da amostra

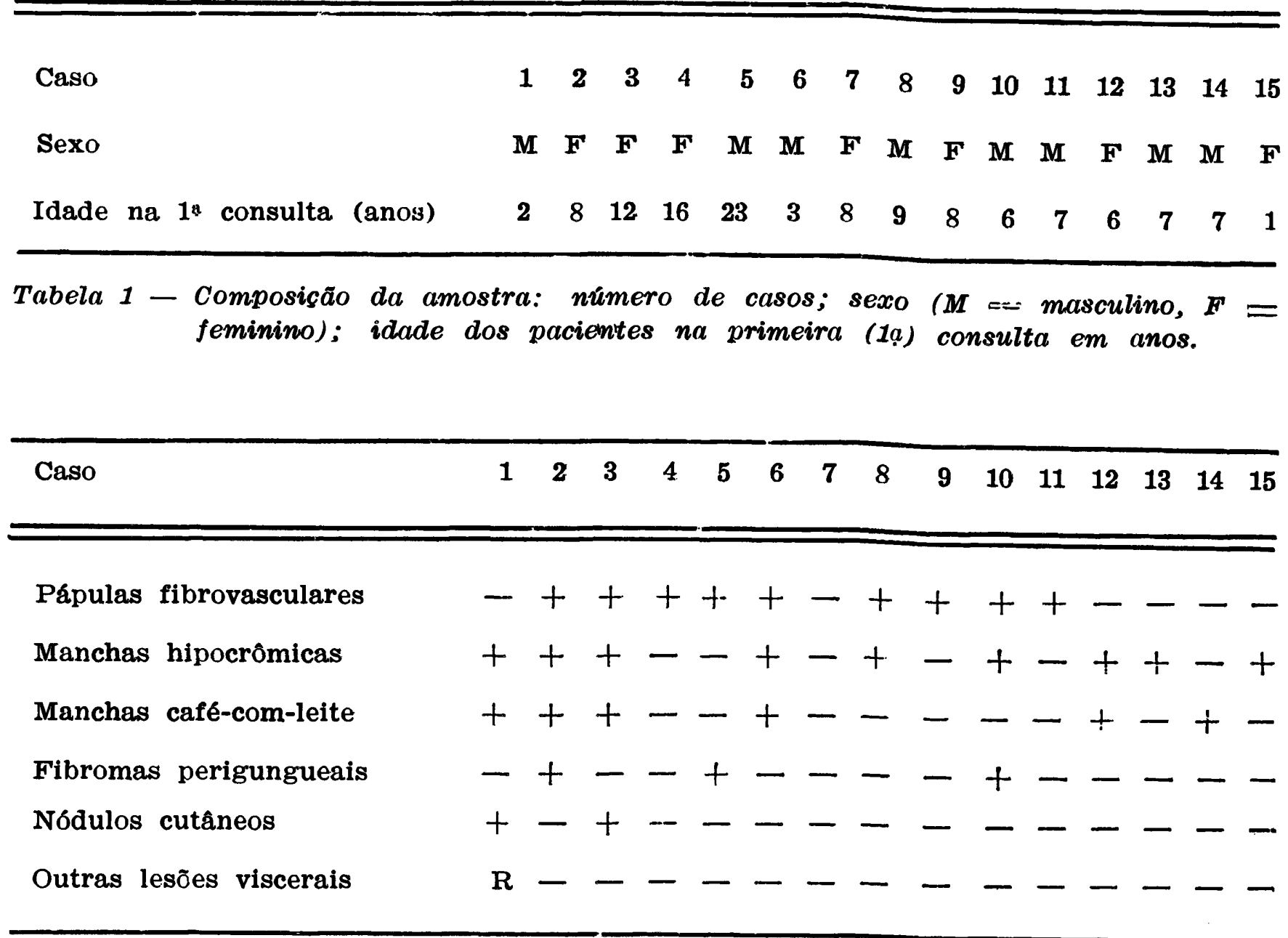

Tabela 2 - Sinais clinicos dos 15 casos de esclerose tuberosa. Legenda: presentes $(-t)$, ausentes (-), rabdomioma (R).

Exames complementares (Tabela 4) - O eletrencefalograma mostrou-se alterado em 8 dos 12 casos em que foi realizado, tendo apresentado aspecto hipsarrítmico em apenas 1 caso. O RX simples de crânio, feito em 6 casos, mostrou calcificações intracranianas em 2 deles. Verificamos ainda que em 4 casos com $R X$ de crânio normal, a tomografia axial computadorizada mostrou grande número de nódulos calcificados de situação periventricular. O exame de fundo de olho, realizado em 8 casos, mostrou facoma retiniano em apenas um caso.

\section{COMENTARIOS}

Sabe-se que o substrato anatômico responsável pela gravidade da doença é constituído principalmente por nódulos gliais displásicos calcificados, situados geralmente ao redor das cavidades ventriculares. Estes puderam ser evidenciados em 7 dos nossos casos. Foram mostrados pela tomografia do crânio em 4 casos, cujos exames radiológicos eram normais. Como já referido por outros autores, a tomodensimetria permite detectar nódulos calcificados bem antes de sua visualização radiológica ${ }^{8}$. 
Entre as manifestações cutâneas, a mais constante foi o adenoma sebáceo, termo usado por Balzer, Ménétrier e Pringle, porém considerado inadequado, porque as lesões não são nem adenomatosas nem sebáceas. Considerando-se sua constituição histológica, estas lesões seriam mais corretamente denominadas pápulas fibrovasculares ${ }^{13}$. Esta lesão cutânea, que pode ser considerada patognomônica da esclerose tuberosa, estava presente em $60 \%$ dos nossos pacientes até a época do último exame clínico, mostrando uma percentagem semelhante à encontrada por outros autores. As pápulas fibrovasculares podem-se desenvolver em qualquer idade, aparecendo principalmente na primeira década da vida. Dos nossos 6 pacientes sem a lesão facial, o mais velho tinha 8 anos de idade.

\begin{tabular}{|c|c|c|c|}
\hline Casos & Nivel Intelectual & $\begin{array}{l}\text { Distúrbios } \\
\text { psíquicos }\end{array}$ & $\begin{array}{l}\text { Crises epilépticas } \\
\text { (Idade do aparecimento) }\end{array}$ \\
\hline 1 & $\begin{array}{l}\mathrm{DM} \text { moderada } \\
\mathrm{QDM} \simeq 38 ; \mathrm{QDA} \simeq 40\end{array}$ & - & $\begin{array}{l}\text { Clônicas generalizadas } \\
\text { (6 meses); tônicas generalizadas }\end{array}$ \\
\hline 2 & $\begin{array}{l}\mathrm{QDL} \sim 29 ; \mathrm{QDS} \simeq 39 \\
\mathrm{DM} \text { moderada } \\
\mathrm{QI}=46\end{array}$ & 一 & $\begin{array}{l}\text { (2 anos); ausências atípicas } \\
\text { Espasmos (dos } 2 \text { meses e meio } \\
\text { aos } 9 \text { meses); hemigeneralizadas; } \\
\text { ausências atípicas }\end{array}$ \\
\hline 3 & $\begin{array}{l}\mathrm{DM} \text { moderada } \\
\mathrm{QI}=47\end{array}$ & Hiperatividade & $\begin{array}{l}\text { Sindrome de West ( } 8 \text { meses); } \\
\text { mioclônicas }\end{array}$ \\
\hline 4 & $\begin{array}{l}\text { DM leve } \\
\mathrm{QI}=56\end{array}$ & - & Tônico-clônicas (3 anos) \\
\hline 5 & $\begin{array}{l}\text { Inteligência limitrofe } \\
\mathrm{QI}=72\end{array}$ & $\begin{array}{l}\text { Agressividade: } \\
\text { alucinações auditivas }\end{array}$ & $\begin{array}{l}\text { Mistas: tônicas, versivas, } \\
\text { ausências ( } 7 \text { meses) }\end{array}$ \\
\hline 6 & $\begin{array}{l}\text { Inteligência limitrofe } \\
\text { QI }=71\end{array}$ & Agressividade & $\begin{array}{l}\text { Atônicas (1 ano); parciais } \\
\text { motoras braquifaciais à } \mathbf{E} \\
(3 \text { anos) }\end{array}$ \\
\hline 7 & DM profunda & Agressividade & $\begin{array}{l}\text { Mistas: ausências, tônicas e } \\
\text { tônico-clônicas }\end{array}$ \\
\hline 8 & DM profunda & Agressividade & Mioclônicas \\
\hline 9 & DM profunda & - & Tônicas \\
\hline 10 & DM profunda & Hiperatividade & Clônicas generalizadas (7 meses) \\
\hline 11 & DM profunda & $\begin{array}{l}\text { Hiperatividade; } \\
\text { agressividade }\end{array}$ & Espasmos infantis \\
\hline 12 & DM profunda & Hiperatividade & - \\
\hline 13 & $\begin{array}{l}\mathrm{DM} \text { moderada } \\
\mathrm{QI}=47\end{array}$ & Hiperatividade & Tônicas \\
\hline 14 & $\begin{array}{l}\text { DM moderada } \\
\mathrm{QI}=5 \mathbf{P}\end{array}$ & - & - \\
\hline 15 & $\begin{array}{l}\text { DM moderada } \\
\text { QDM } \simeq 37 ; \mathrm{QDA}=42 \\
\mathrm{QDS} \simeq 2 \mathrm{\sim} 26 \mathrm{QDL}=27\end{array}$ & 一 & $\begin{array}{l}\text { Espasmos; ausências atípicas; } \\
\text { hemigeneralizadas clônicas }\end{array}$ \\
\hline
\end{tabular}

Tabela : - Sintomatologia neuro-psiquiatrica. Legenda: deficiência mental (DM), quociente de desenvolvimento motor (QDM), quociente de desenvolvimento adaptativo (QDA), quociente de desenvolvimento da linguagem (QDL), quociemte de desenvolvimento social (QDS), quociente intelectual (QI), esquerda (E). 


\begin{tabular}{|c|c|c|c|c|}
\hline Casos & Fundoscopia & RX Crânio & TAC & EEG \\
\hline 1 & $\mathbf{N}$ & $\mathbf{N}$ & CPV & AP difusa \\
\hline 2 & $\mathbf{N}$ & $\mathbf{C}$ & CPV & AP centro parieto-temporal $\mathrm{D}$ \\
\hline 3 & $\begin{array}{l}\text { Facoma no } \\
\text { olho } \mathrm{E}\end{array}$ & $\mathbf{N}$ & CPV & 1 - hipsarritmia \\
\hline 4 & - & - & - & 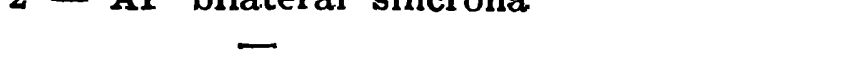 \\
\hline 5 & - & - & - & AI frontal $D$ \\
\hline 6 & $\mathbf{N}$ & $\mathbf{N}$ & CPV & AI de projeção centro-parietal $D$ \\
\hline 7 & 一 & - & - & Pobremente organizado com surtos difusos \\
\hline 8 & $\mathbf{N}$ & $\mathbf{C}$ & CPV & AP difusa \\
\hline 9 & - & - & - & - \\
\hline 10 & $\mathbf{N}$ & - & - & Lento para a idade \\
\hline 11 & - & - & - & $\bar{\pi}$ \\
\hline 12 & - & - & - & $\mathbf{N}$ \\
\hline 13 & $\mathbf{N}$ & - & CPV & $\mathbf{N}$ \\
\hline 14 & - & - & - & $\mathbf{N}$ \\
\hline 15 & $\mathbf{N}$ & $\mathbf{N}$ & CPV & $\mathbf{N}$ \\
\hline
\end{tabular}

Tabela 4 - Exames complementares: fundoscopia, $R X$ simples de cranio (RX cranio), tomografia axial computadorizada (TAC), eletrencefalograma (EEG). Legenda: exame não realizado (-), exame normal ou sem anormalidades (N), lado esquerdo (E), lado direito (D), calcificaçoses $(C)$, calcificaçós periventriculares (CPV), anormalidade paroxistica (AP), anormalidade irritativa $(A I)$.

Do total de casos, em $60 \%$ havia manchas hipocrônicas, consideradas muito importantes para o diagnóstico precoce da esclerose tuberosa e aparecendo, na maioria dos casos, em torno do segundo ano de vida 4. A importância da presença de máculas hipopigmentadas para o diagnóstico precoce da esclerose tuberosa foi sugerida por Hurwitz e Bravermans, que as encontraram em 78\% dos seus pacientes. No caso 15 da nossa amostra, o diagnóstico foi suspeitado pela presença de manchas hipocrônicas e epilepsia.

Os principais sintomas neurológicos foram a deficiência mental (DM) e a epilepsia. $\mathrm{Na}$ literatura mais antiga, encontramos referência praticamente constante à deficiência mental como parte essencial do quadro clínico, uma vez que a maioria dos casos provinha de instituições especializadas em deficiência mental. Mais recentemente, Lagos e Gomez ${ }^{9}$ relataram 71 casos da Clínica Mayo, com uma incidência de 62\% de DM. Pou-Serradell e col. ${ }^{15}$ a encontraram em $85 \%$ e Pampiglione e col. ${ }^{14} \mathrm{em} 88 \%$ de seus casos. Dos nossos pacientes, $86 \%$ apresentavam deficiência mental. Parece não haver correlação entre 0 déficit intelectual e a gravidade da epilepsia ${ }^{15}$. Dos nossos 2 pacierites que não apresentavam epilepsia, um era deficiente mental profundo e o outro, moderado.

A manifestação neurológica mais importante e, na maioria dos casos relatados na literatura, o motivo da primeira consulta médica, são as crises epilépticas. Como manifestação precoce, destaca-se a sindrome dos espasmos infantis, relacionados à esclerose tuberosa, pela primeira vez, em 1959 10. Vários autores, como Fois ${ }^{4}$ e Pou-Serradell ${ }^{15}$, ressaltam a importância das máculas hipo- 
crômicas associadas aos espasmos infantis para o diagnóstico precoce da esclerose tuberosa. A associação de espasmos em flexão e manchas acrômicas (no mínimo 3) é patognomônica de esclerose tuberosa ${ }^{15}$. Quatro de nossos 13 pacientes epilépticos apresentavam espasmos infantis no primeiro ano de vida e em um deles (caso 3), o traçado eletrencefalográfico teve aspecto hipsarrítmico. Ainda em relação às crises, ressaltamos o grande número de pacientes com epilepsia mista e de difícil controle medicamentoso, enfatizando a presença de crises tônicas e ausências atípicas.

Entre as manifestações psiquiátricas, encontramos hiperatividade em 5 casos (33\%), agressividade em 5 casos (33\%) e, em um dos casos, distúrbios senso-perceptivos, especificamente alucinações auditivas, que, juntamente com o restante do exame psiquiátrico, levaram ao diagnóstico de psicose epiléptica.

Alguns autores ${ }^{3}$ sugerem que a hiperatividade em geral seja eminentemente de base orgânica, sendo inclusive mais frequentemente verificada em portadores de deficiência intelectual, da mesma forma que consideram que as crianças hiperativas apresentam outros disturbios de conduta, entre eles impulsividade e agressividade. Observando os nossos achados, embora a etiologia desta hiperatividade seja de difícil caracterização, ela nos parece independente da gravidade da DM, visto ter sido encontrada em seus mais variados graus (Tabela 3). Por outro lado, quando consideramos as manifestações psíquicas de linhagem epiléptica ${ }^{11}$, vemos que fazem parte de seu cortejo sintomatológico a impulsividade, a agressividade e, em algumas ocasiões, os quadros psicóticos de evolução em surtos com colorido esquizomorfo e remissão espontânea em muitas ocasiões, a exemplo do caso descrito anteriormente. Portanto, parece-nos justo considerar também tais alterações psíquicas como decorrentes do quadro orgânico de base.

Deve ser mencionado ainda que, no caso 1, o diagnóstico de esclerose tuberosa foi feito a partir do achado de um rabdomioma cardíaco, numa época em que as outras manifestações da doença ainda não tinham aparecido. A esclerose tuberosa é doença de hamartomas múltiplos de origem ectodérmica e mesodérmica ${ }^{6}$. Assim, podem ocorrer angiomiolipomas renais, rabdomiomas cardíacos, cistos pulmonares, lesões císticas ósseas, lesões hamartomatosas em fígado, tireóide, testículos e baço.

Quanto à sua etiologia, a esclerose tuberosa é uma doença determinada por gene autossômico dominante 2,12 de expressividade variável. Nosso estudo vem ao encontro da hipótese de herança dominante simples. Dos 15 pacientes estudados, 11 (73\%) foram considerados como exemplos de mutações novas, visto que a história familiar foi negativa $e$ os pais não apresentavam evidência clinica da esclerose tuberosa. Uma paciente (caso 2) é filha adotiva, não existindo informações sobre sua família. Em 3 pacientes (casos 5, 11 e 12), não foi possivel obter dados sobre os familiares dos pacientes. Nas publicações sobre esclerose tuberosa, poucos casos foram considerados familiares, a maioria (cerca de 80 a $85 \%$ ) sendo dados como decorrentes de mutações novas do mesmo locus gênico. 


\section{RESUMO}

São apresentados os resultados de estudo multidisciplinar de 15 pacientes com esclerose tuberosa, 8 do sexo masculino e 7 do sexo feminino, com idades entre 19 meses e 23 anos; 11 foram considerados como casos esporádicos e, enı 4, não foi possivel precisar os antecedentes familiares. Foram observadas as seguintes manifestações cutâneas: adenoma de Pringle em 9 casos; manchas acrômicas em 9 casos; fibroma periungueal em 3 casos. Dos 15 casos 13 apresentaram D.M., deficiência mental, enquanto os outros 2 tinham inteligência limítrofe. Com exceção de 2 pacientes, todos tinham crises epilépticas e os tipos de crises mais frequentemente observados foram as ausências atípicas $\mathrm{e}$ as crises tônicas. Espasmos infantis estavam presentes em 3 casos e em apenas um caso o eletrencefalograma apresentou aspecto hipsarrítmico. A tomografia axial computadorizada, realizada em 7 casos, revelou calcificações periventriculares em todos, mesmo naqueles que apresentaram RX simples de crânio normal (4 casos).

\section{SUMMARY}

Tuberous sclerosis: a multidisciplinary study of 15 cases.

Report of a multidisciplinary study of patients affected with tuberous sclerosis, 8 of them males and 7 females, with ages ranging from 19 months to 23 years; 11 of these cases were sporadic, while the remaining 4 cases lacked information regarding family data. The following skin signs were observed: Pringle's adenoma in 9 cases; achromatic spots in 9 cases; periungueal fibroma in 3 cases. One of the patients had a heart tumor. Two patients had borderline intelligence, while the other 13 were mentally retarded of varying degrees. Epileptic seizures were present in 13 patients, atypical absence and tonus crises were frequent. Three of the patients had had infantile spasms in the first year of life as the first neurological symptom of the disease. Computer assisted axial tomography was performed in 7 cases and was positive in all of them, by revealing intracranial calcifications even in 4 cases in which the standard $X$

- ray pictures had been silent about this eventuality.

\section{REFERENCIAS}

1. BERGsma, D. - Birth Defects Compendium. Second Edition. Alon. R. Siss Inc., New York, 1979, pg. 1.053.

2. BUNDEY, S. \& EVANS, K. - Tuberous sclerosis: a genetic study. J. Neurol. Neurosurg. Psychiat. 32:591, 1969.

3. CHESS, S. - Diagnosis and treatment of the hyperative child. J. Med. 60:2379, 1960.

4. FOIS, A.; PINDINELLI, C. A. \& BERARDI, R. - Early signs of tuberous sclerosis in infancy and childhood. Helv. paediat. Acta 28:313, 1973.

5. HURWITZ, S. \& BRAVRRMAN, I. M. - White spots in tuberous sclerosis. J. Ped. 77:587, 1970.

6. JONES, J. C.; MONTOYA, JR, S. F. \& JOHNSON, J. W. - Tuberous sclerosis presenting as chronic renal failure. South. Med. J. 73:551, 1980. 
7. KAPP, J. P.; PAULSON, G. W. \& ODOM, G. L. - Brain tumors with tuberous sclerosis. J. Neurosurg. 26:191, 1967.

8. KAZNER, E.; LANKSCH, W. \& STEINHOFF, H. - Cranial computerized tomography in the diagnosis of brain disorders in infant and children. Neuropaediat. 7:136, 1976.

9. LAGOS, J. C. \& GOMEZ, M. R. - Tuberous sclerosis: reappraisal of a clinical entity. Mayo Clin. Proc. 42:26, 1967.

10. MACCAGNANI, F. \& VIZIOLI, R. - Considerazioni sulla patogenesi degli spasmi infantili alla luce di un caso operato di un nodulo di sclerosi tuberosa ad evoluzione neoplastica situato nella testa del nucleo cat-sado. Riv. Neurol. (Roma) 29:897, 1959.

11. Matarazzo, E. B. - Psicose epiléptica na infância e na adolescência. Tese. São Paulo, 1977.

12. McKUSIK, V. A. - Mendelian inheritance in man. Catalogs of autosomal dominant, autosomal recessive and $\mathrm{X}$ - linked phenotypes. Johns Hopkins Univ. Press. Baltimore, 1978, pg. 379.

13. MORGAN, J. E. \& WOLFORT, F. - The early history of tuberous sclerosis. Arch. Dermatol. 115:1317, 1979.

14. PAMPIGLIONE, G. \& MOYNAHAN, E. J. - The tuberous sclerosis syndrome: clinical and EEG studies in 100 children. J. Neurol. Neurosurg. Psychiat. 39:666, 1976.

15. POU-SERRADELL, A.; PASCUAL J.; UGARTE, A.; FlORENSA, R. \& SOLER, L. - Etude longitudinale de 14 cas de sclérose tubéreuse de Bourneville. Rev. neurol. (Paris) 135:693, 1979.

16. ROTH, J. C. \& EPSTEIN, C. J. - Infantile spasm and hypopigmented maculs. Arch. Neurol. (Chicago) 25:547, 1971.

APAE - Associação de Pais e Amigos dos Excepcionais - Centro de Habilitação - Caixa Postal 20369 - 01000 São Paulo, SP - Brasil. 